Nouvelles perspectives en sciences sociales

Revue internationale de systémique complexe et d'études relationnelles

\title{
Se différencier ou se conformer : enjeux de la recherche en sociologie sur les cultures juvéniles, enjeux des cultures juvéniles...
}

\section{Sylvain Aquatias}

Volume 8, numéro 1, novembre 2012

Sur le thème de l'homogénéisation et de la différenciation

URI : https://id.erudit.org/iderudit/1013919ar

DOI : https://doi.org/10.7202/1013919ar

Aller au sommaire du numéro

\section{Éditeur(s)}

Prise de parole

ISSN

1712-8307 (imprimé)

1918-7475 (numérique)

Découvrir la revue

Citer cet article

Aquatias, S. (2012). Se différencier ou se conformer : enjeux de la recherche en sociologie sur les cultures juvéniles, enjeux des cultures juvéniles.... Nouvelles perspectives en sciences sociales, 8(1), 83-117. https://doi.org/10.7202/1013919ar

\section{Résumé de l'article}

Depuis quelques années, les sociologues qui étudient les cultures juvéniles s'opposent : d'un côté, on décrit un ensemble tendant à s'homogénéiser par la puissance des normes adolescentes ou à s'hétérogénéiser du fait d'un d'éclectisme généralisé, de l'autre on réaffirme la force primordiale des socialisations familiales et scolaires et des effets de distinction des goûts culturels. Les résultats de la recherche présentée ici montrent que c'est dans l'action conjointe des situations familiales, des parcours scolaires et des réseaux amicaux que se créent des zones d'influence plus ou moins puissantes, déterminées en grande partie par la cohésion sociale des différentes instances en présence. Ainsi, la qualité des relations avec les parents, les modes de scolarisation, les filières et la cohésion interne des groupes classes sont d'importants facteurs d'explication d'une emprise plus ou moins durable sur les goûts adolescents. Conformité et distinction prennent sens dans ces configurations d'influences. 


\title{
Se différencier ou se conformer : enjeux de la recherche en sociologie sur les cultures juvéniles, enjeux des cultures juvéniles...
}

Sylvain AQuatias
Groupe de recherches et d'études sociologiques du Centre-Ouest

(GRESCO)

\begin{abstract}
Contre l'idée, longtemps bien partagée, d'une jeunesse malléable et influençable, dominée par la force des institutions familiales et scolaires, s'est affirmée depuis une dizaine d'années, non seulement l'idée d'une plus grande autonomie des goûts adolescents, mais plus encore celle d'une culture juvénile relativement singulière par rapport aux autres classes d'âge.

L'avènement de l'influence des pairs a un fort impact dans ce débat. Depuis les écrits de Judith Harris ${ }^{1}$, l'on n'a pas cessé de mettre en avant la manière dont les jeunes, entre eux, pouvaient faire émerger des valeurs contraires à celles défendues par les générations antérieures. S’y ajoute le développement des médias et des nouvelles technologies. Les représentations actuelles de l'adolescence appréhendent la jeunesse soit comme passive et soumise aux influences médiatiques, soit comme constituée de consommateurs aguerris et critiques ${ }^{2}$.
\end{abstract}

\footnotetext{
1 Judith Harris, Pourquoi nos enfants deviennent ce qu'ils sont, Paris, Laffont, 1999.

2 David Buckingham, La Mort de l'enfance, grandir à l'âge des médias, Paris, Armand Colin, 2000.
} 
Dans ce contexte de transformations sociales, les courants sociologiques s'opposent. Soit l'on met l'accent sur ce qui réunit les jeunes, dans un mouvement centripète de conformisme qui les amène à se ressembler, soit on les présente comme des individus en construction, leurs goûts se diversifiant en même temps qu'ils connaissent des expériences diverses. Hétérogénéisation et homogénéisation constituent ici deux pôles opposés des manières d'aborder les cultures juvéniles.

C'est la définition même de l'adolescence - et donc de la culture juvénile - qui se joue alors, selon qu'on considère qu'elle se forme à partir de multiples éléments ou qu'elle est dominée par un facteur d'influence majeur, qu'il s'agisse des transmissions familiales et/ou scolaires ou des normes adolescentes. Plus encore, le fait de concevoir la jeunesse comme étant homogène ou hétérogène dépasse la simple question des cultures juvéniles pour aborder de manière plus générale les processus de légitimation des biens culturels et leurs liens avec la socialisation. C'est donc un débat complexe qui se noue autour de cette question de la culture juvénile, débat que l'on ne peut aborder sans en restituer les enjeux scientifiques.

\section{Légitimité, éclectisme et affinités}

Car l'opposition entre chercheurs se construit d'abord autour de relectures de la théorie de la légitimité, qui fait correspondre goûts et positions sociales, choix culturels et effets de distinction, influences parentales et scolaires et socialisation ${ }^{3}$. Cette théorie a permis, d'une part, d'expliquer les écarts significatifs dans la distribution des consommations culturelles des Français par l'appartenance à différents milieux sociaux et, d'autre part, de décrire le processus de hiérarchisation des productions culturelles, légitimées par les classes dominantes. Elle est actuellement remise en cause, tant de manière globale qu'en ce qui concerne des publics plus précis, comme les adolescents.

$\overline{3}$ Pierre Bourdieu, La Distinction. Critique du jugement, Paris, Minuit, 1979. 
Probablement sont-ce les travaux d'Olivier Donnat qui ont ouvert la brèche en postulant une plus grande homogénéisation des cultures juvéniles :

Les adolescents, notamment, présentent, à l'échelle de la population française, une configuration particulière de compétences, comportements et préférences culturelles qui constituent un ensemble de traits suffisamment stables et cohérents pour les distinguer du reste de la population. Dans ce cas, pourquoi ne pas parler de « culture jeune » au même titre qu'on parle de "culture cultivée " pour désigner les activités et les goûts caractéristiques des milieux diplômés? ${ }^{4}$

La différence qui s'élabore alors entre classes d'âge semble dépasser les déterminismes des origines sociales. Plus fondamentalement, c'est au dépassement de l'opposition entre des productions médiatiques considérées comme illégitimes et une culture savante légitimée par l'École et l'État qu'appellent Éric Maigret et Éric Macé, en évoquant « des médiacultures, points d'intersection des phénomènes démocratiques contemporains de construction du sens et de la valeur $[\ldots]^{5}$ ". Ce renouvellement du champ est en partie lié au développement des nouvelles technologies de l'information et de la communication et à la multiplication des sources d'influence qu'elles produisent. L'ouvrage qu'ils dirigent en appelle très clairement à " la fin du modèle classique de la légitimité culturelle ${ }^{6} »$.

Face à ces arguments, les défenseurs de la théorie de la légitimité développent principalement deux arguments. C'est d'abord d'une relative permanence des déterminismes sociaux qu'il est question; encore à présent, certaines pratiques ne peuvent se comprendre en dehors de ce schéma ${ }^{7}$. C'est ensuite la reconstruction des hiérarchies de distinction qui est avancée : aux champs habituellement séparés de la culture légitime et de la culture

$4 \quad$ Olivier Donnat (dir.), Regards croisés sur les pratiques culturelles, Paris, La documentation française, 2003, p. 16.

5 Éric Maigret et Éric Macé (dir), Penser les médiacultures, Paris, Armand Colin, 2006, p. 10.

6 Ibid, p. 69. C'est le titre du chapitre 3, écrit par Hervé Glevarec, p. 69-102.

7 C'est « l'introuvable moyennisation des pratiques et des attitudes culturelles " dont parle Philippe Coulangeon dans Les Métamorphoses de la distinction, Paris, Grasset, 2011, p. 28. 
populaire, se substitue alors une opposition entre les univores (ceux dont les goûts sont peu diversifiés et qui sont plutôt issus des classes populaires) et les omnivores (ceux qui aiment de multiples genres, aussi bien populaires que savants, plutôt issus des classes favorisées), faisant référence aux travaux de Richard Peterson ${ }^{8}$.

Que ce soit du côté de ceux qui attaquent le modèle bourdieusien ou de ceux qui le défendent, les arguments sont fondés. On trouve aussi bien des permanences, notamment dans la fréquentation des musées ou la lecture, qu'un déploiement des références culturelles populaires, entre autres dans le domaine musical. C'est par ailleurs de ce dernier exemple que se servent Hervé Glévarec et Michel Pinet pour appeler à un changement de paradigme?, en remettant en cause l'interprétation qui établit un lien entre omnivorité et classes supérieures. Les auteurs ne s'attachent pas aux seules cultures juvéniles, mais s'appuient souvent sur elles pour nourrir leur démonstration. C'est même ainsi qu'ils assoient leur raisonnement, en montrant combien les goûts musicaux des jeunes générations sont marqués par les courants populaires.

On peut opposer quelques objections à ce raisonnement. D'une part, s'il est clair que, dans le domaine de l'écoute musicale, les goûts semblent s'être libérés au moins en partie des déterminismes sociaux, il est difficile d'en déduire que c'est le cas dans tous les domaines culturels. D'autre part, les arguments des opposants à la théorie de la légitimité se contredisent parfois. Ainsi, la culture juvénile apparaît-elle chez Dominique Pasquier comme un ensemble tendant à s'homogénéiser par la puissance

8 Richard A. Peterson et Alfred Simkus, "How Musical Tastes Mark Occupationnal Status Groups ", dans Michèle Lamont et Marcel Fournier (dir.), Cultivating Differences, Symbolic Boundaries and the Making of Inequality, Chicago, Londres, University of Chicago Press, 1992, p. 152-186. Voir, pour l'interprétation française de cette théorie, Olivier Donnat, Les Français face à la culture. De l'exclusion à l'éclectisme, Paris, La Découverte, 1994, et Wenceslas Lizé et Olivier Roueff, "La Fabrique des goûts ", Actes de la recherche en sciences sociales, $\mathrm{n}^{\text {os }}$ 181-182, mars 2010, p. 4-11.

9 Hervé Glevarec et Michel Pinet, "La "Tablature" des goûts musicaux : un modèle de structuration des préférences et des jugements ", Revue française de sociologie, vol. 50, $\mathrm{n}^{\circ}$ 3, 2009, p. 599-640. 
des normes adolescentes ${ }^{10}$, s'opposant en cela d'une part au constat qui, porté par les dernières grandes enquêtes nationales sur les pratiques culturelles des Français, établit que la jeunesse est l'âge où la diversité des références est la plus forte ${ }^{11}$, et d'autre part aux propositions d'Hervé Glevarec et de Michel Pinet, qui postulent que le rapport social qui guide les préférences musicales n'est plus la distinction ou le rejet mais l'ouverture ou la tolérance $^{12}$. Ces hypothèses ne sont pas totalement antinomiques : en effet, on peut considérer que l'effet normalisateur des groupes de jeunes n'agit que dans le cadre des sociabilités qu'ils partagent et que les goûts les moins admissibles sont nourris dans l'intimité. Mais cela nécessite que les pratiques soient relativement cloisonnées, ce qui est délicat à un âge où le développement des relations sociales tient une grande place et où trop s'écarter des modèles admis pourrait avoir pour conséquence d'être exclu des groupes de pairs ${ }^{13}$.

Quoi qu'il en soit, le simple fait d'admettre que les influences amicales jouent un rôle majeur sur les goûts adolescents s'oppose à la pensée bourdieusienne en ce sens que cette dernière a surtout conceptualisé la socialisation à travers le rôle des grandes institutions (école, famille) et non à travers les relations quotidiennes des acteurs. Les chercheurs qui condamnent le rôle des médias, lesquels influenceraient négativement les adolescents, ne font pas autre chose, d'une certaine manière, que de soutenir cette idée, puisque les jeunes n'apparaissent alors plus comme des entités autonomes, mais bien comme les victimes de programmes profitant de la malléabilité juvénile ${ }^{14}$. Pourtant, s'il est indéniable que les médias peuvent avoir un effet sur leurs publics, il est tout aussi impossible de ne pas concevoir que les consommateurs puissent disposer d'au moins certaines facultés critiques. Le débat

10 Dominique Pasquier, Cultures lycéennes, la tyrannie de la majorité, Paris, Autrement, 2005.

11 Bernard Lahire, La Culture des individus. Dossonnances culturelles et distinction de soi, Paris, La Découverte, 2004, p. 205.

12 Hervé Glevarec et Michel Pinet, op. cit., p. 624.

13 Dominique Pasquier, «La Culture comme activité sociale », dans Éric Maigret et Éric Macé (dir.), Penser les médiacultures, op. cit., p. 103-119.

14 David Buckingham, op. cit. 
sur la plus ou moins grande autonomie des jeunes en matière de goûts culturels repose alors essentiellement sur la perception que l'on aura des hommes en société, agents ou acteurs, reposant une opposition fondamentale de la sociologie.

On le voit, deux points de vue s'affrontent, celui de l'homogénéité (Dominique Pasquier et Hervé Glevarec défendent le postulat d'une jeunesse aux goûts plus homogènes), celui de l'hétérogénéité (la tradition bourdieusienne, bien révélée par le titre d'un entretien de Pierre Bourdieu, "La jeunesse n'est qu'un $\left.\operatorname{mot}^{15} »\right)$. Mais, alors que le structuralisme génétique postule une relative homogénéité d'influences (milieu social et école), ses opposants défendent un modèle affinitaire où " le rapport à la culture n'est pas uniquement dessiné par les origines culturelles et sociales, il est aussi dicté par les bifurcations biographiques et l'environnement social ${ }^{16}$ ". Hétérogénéité des goûts et relative homogénéité des influences d'un côté, homogénéité des goûts et hétérogénéité des influences de l'autre, voilà qui complexifie encore le problème.

Cet objet de recherche pourrait être examiné dans les conditions mêmes de l'analyse bourdieusienne, comme une tentative de se situer dans le champ sociologique, en fonction des théories dominantes. Et l'étalon est bien ici la théorie de la légitimité, que l'on cherche à la sauvegarder ou à la mettre à mal. La sociologie n'échappe pas aux enjeux des positionnements scientifiques ${ }^{17}$. Au demeurant, les cultures juvéniles n'ont jamais bénéficié d'une grande légitimité au sein des travaux sociologiques et, comme telles, constituent alors un domaine de pensée plus libre et plus ouvert à l'innovation théorique ${ }^{18}$. On comprend dès lors que les positionnements des chercheurs créent des antagonismes, selon qu'ils s'inscrivent en conformité avec ce qui apparaît être le

15 Pierre Bourdieu, Questions de sociologie, Minuit, Paris, 1984, p. 143-154.

16 Dominique Pasquier, "La Culture comme activité sociale ", op. cit., p. 107.

17 Pierre Bourdieu, «La Spécificité du champ scientifique et les conditions sociales du progrès de la raison ", Sociologie et sociétés, vol. 7, $\mathrm{n}^{\circ} 1,1975$, p. 91-118.

18 Sylvie Octobre et al, L'Enfance des Loisirs. Trajectoires communes et parcours individuels de la fin de l'enfance à la grande adolescence, Ministère de la Culture, Paris, 2010, p. 13-22. 
modèle dominant ou en opposition avec celui-ci. Et pourtant, on l'a dit, les arguments sont fondés des deux côtés. Dans ce cadre, le seul parti raisonnable est de partir du terrain pour examiner ces théories à l'aune des pratiques.

C'est ce que je vais essayer de faire à présent, en examinant les différentes influences, celles qui poussent à la ressemblance et celles qui motivent la différenciation, à l'aide des données d'une recherche effectuée dans le Limousin, portant sur les goûts juvéniles et articulant données quantitatives et qualitatives.

L'enquête quantitative portait sur les activités de loisirs des jeunes scolarisés, elle a été menée en 2009 auprès d'une population représentative d'élèves du Limousin (Rapport téléchargeable sur www.limousin.iufm.fr). Léchantillon a été construit par la méthode des quotas en suivant plusieurs critères établis grâce aux chiffres fournis par les services rectoraux : d'abord au niveau des grandes caractéristiques des établissements (collège, lycée général et technologique, lycée professionnel, milieu rural ou urbain), puis au niveau des élèves présents dans ces établissements (effectif, classe et sexe). C'est au total 1114 questionnaires qui ont été validés, provenant de cinquante classes réparties proportionnellement aux effectifs régionaux (tirage à $5 \%$ ) entre cinquième (20 classes, 439 élèves), troisième (15 classes, 326 élèves), première générale et technologique (10 classes, 247 élèves) et lycée professionnel ( 5 classes, 102 élèves) afin de mieux percevoir l'évolution des intérêts culturels. L'effet des groupes classes a été mesuré et certaines questions permettent de saisir les influences des goûts des élèves.

Cette enquête diffère des statistiques employées par les enquêtes d'Olivier Donnat, de Sylvie Octobre, de Philippe Coulangeon ou d'Hervé Glevarec qui se basent sur des analyses en régression logistique. Ces résultats ne sont donc pas de même nature. Néanmoins, leurs convergences permettent de les relier positivement : globalement, nos résultats régionaux retranscrivent les tendances décrites par les enquêtes nationales.

L'enquête qualitative correspond à une vague d'entretiens avec des jeunes nés en 1994, soit en filière générale, soit en filière 
professionnelle, garçons et filles, portant sur la construction des goûts (vingt-deux entretiens). L'entretien portait sur les activités de loisirs et ce qui les influençait, en insistant sur les loisirs les plus importants au regard des jeunes. La prise de contact avec les élèves s'est faite par le biais des enseignants et des conseillers principaux d'éducation, ce qui implique un léger biais au niveau des jeunes qui sont, en lycée général, souvent de bons élèves, ce qui explique aussi la relative disproportion entre classes favorisées (9), moyennes (9) et défavorisées (4).

La vague d'entretiens concerne principalement les élèves en cursus général. Des entretiens complémentaires en lycée professionnel sont en cours de réalisation. Les 22 entretiens qui ont nourri l'analyse se décomposent en 10 garçons (dont 2 en lycée professionnel) et 12 filles (dont 3 en lycée professionnel).

On traitera ici de lecture et d'écoute de musique. D'une part, la lecture est encore un des domaines culturels où perdurent les influences familiales et scolaires, d'autre part, l'écoute musicale paraît au contraire s'être affranchie de ces deux institutions. En confrontant ces deux domaines, nous pourrons mieux saisir les différents agents d'influences et leurs arbitrages.

L'analyse proposée ici repose sur le croisement des données quantitatives et qualitatives. Elle permet de compenser la relative déperdition d'informations que l'analyse quantitative seule provoque $^{19}$ : on ne peut alors saisir le substrat des influences et des choix. La continuité d'analyse proposée ici, entre les goûts et les phénomènes d'affirmation de soi, n'est rendue possible que par cette articulation.

Il existe une forte conjonction entre l'âge et la classe dans l'échantillon quantitatif : 84,5\% des élèves de cinquième ont 12 ou 13 ans, $78 \%$ des élèves de troisième ont 14 ou 15 ans, $75 \%$ des élèves de lycée général et technologique ont 16 ou 17 ans et $76,5 \%$ des élèves de lycée professionnel ont entre 18 et 20 ans. Aussi vais-je présenter ces résultats par classe, pour mieux montrer les influences de la scolarité.

19 Jean-Claude Combessie, La Méthode en sociologie, La Découverte, Paris, 2007, chapitre III. 


\section{De l'homogénéité à la diversification, de la cinquième à la troisième...}

L'évolution des loisirs juvéniles est d'abord liée à l'âge, ce que démontre bien l'enquête longitudinale menée par Sylvie Octobre $^{20}$. C'est aussi, dans notre recherche, ce qui sépare le mieux les élèves, une véritable frontière s'établissant entre les moins de 16 ans et les plus de 16 ans, entre d'un côté les influences familiales et médiatiques, de l'autre les influences des pairs, comme le montrent les quelques indicateurs synthétiques du tableau ci-dessous.

Tableau 1

Quelques indicateurs synthétiques des changements d'influence entre moins de 16 ans et les 16 ans et plus

\begin{tabular}{|c|c|c|c|c|c|c|}
\hline Âge & $\begin{array}{c}\text { Influence } \\
\text { des parents } \\
\text { dans le } \\
\text { choix d'un } \\
\text { livre }\end{array}$ & $\begin{array}{c}\text { Sortie au } \\
\text { cinéma } \\
\text { avec les } \\
\text { parents }\end{array}$ & $\begin{array}{c}\text { Écoute } \\
\text { quotidienne } \\
\text { de la radio }\end{array}$ & $\begin{array}{c}\text { Choix } \\
\text { vestimentaire } \\
\text { influencé par } \\
\text { des chanteurs } \\
\text { ou groupes }\end{array}$ & $\begin{array}{c}\text { Sociabilités } \\
\text { amicales } \\
\text { citées parmi } \\
\text { les } \\
\text { principales } \\
\text { activités de } \\
\text { temps libre }\end{array}$ & $\begin{array}{c}\text { Sortie } \\
\text { avec des } \\
\text { amis* }\end{array}$ \\
\hline $\begin{array}{c}13-15 \\
\text { ans }\end{array}$ & $14,5 \%$ & $47,4 \%$ & $54,9 \%$ & $15,8 \%$ & $7,5 \%$ & $50,3 \%$ \\
\hline $\begin{array}{c}16 \text { ans } \\
\text { et plus }\end{array}$ & $11,3 \%$ & $22 \%$ & $47,9 \%$ & $10,5 \%$ & $15,7 \%$ & $74,7 \%$ \\
\hline
\end{tabular}

Lecture du tableau : c'est 14,5\% des 13-15 ans qui déclarent être influencés par leurs parents dans le choix d'un livre, ce ne sont que $11,3 \%$ des 16 ans et plus.

* Pour toute autre sortie que cinéma, théâtre, concert et musées

Ainsi, ce qui apparaît le plus clairement au niveau de la classe de cinquième, c'est un faible détachement des goûts personnels par rapport aux influences familiales et médiatiques.

Les choix de lecture des élèves de cinquième sont moins diversifiés que ceux de leurs aînés : ainsi, si l'on additionne les trois genres de livres les plus lus par les élèves de cinquième, on obtient $57 \%$ des ouvrages lus. Le même calcul fait pour les élèves

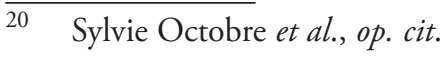


de troisième ne donne qu'un pourcentage de $48 \%$. La gamme des genres lus ne s'est pourtant pas diversifiée, ce sont les ouvrages lus qui se répartissent différemment, ce changement profitant notamment à la littérature contemporaine et à la littérature d'origine scolaire ${ }^{21}$. C'est probablement à ce niveau que commencent à se déterminer des changements de goût qui, selon leur proximité aux canons scolaires, joueront ensuite sur les orientations. Mais, dès la cinquième, les influences de lecture sont bien multiples. L'intérêt personnel et les médias (journaux, télévision, radio et Internet) sont cités par $19 \%$ des élèves, les enseignants comme les parents par $16 \%$ d'entre eux, les amis par $13 \%$.

De même, alors que les élèves de cinquième sont les plus nombreux à déclarer ne pas écouter souvent de musique (26\% contre $19 \%$ des élèves de troisième), ils sont aussi ceux pour qui apparaît un engouement massif pour certains artistes. Ainsi les trois artistes les plus cités regroupent $20 \%$ des élèves de cinquième (contre $13 \%$ des élèves de troisième). Ils sont aussi ceux qui écoutent le plus les mêmes radios, en général des stations musicales, qui représentent $78 \%$ de leurs choix (contre $71 \% \mathrm{du}$ choix des élèves de troisième). L'élévation de l'intérêt pour l'écoute musicale transparaît, pour les élèves de troisième, dans l'augmentation du temps consacré à cette activité (plus du tiers d'entre eux déclare passer au moins une heure par jour à écouter de la musique sans rien faire d'autre), mais c'est aussi un déplacement dans les goûts qui se produit, les élèves appréciant plus les musiques rock au détriment des musiques hip-hop. Par rapport aux autres classes, la troisième est celle où il est le plus difficile de déterminer une relation nette avec un genre musical précis, alors que, dans toutes les autres classes, des goûts se détachent davantage. Il est probable que, le goût musical commençant à se former, les explorations dans des styles multiples sont plus importantes, rendant la stabilisation sur un seul genre improbable.

21 Cette dénomination regroupe les ouvrages dont la lecture découle très probablement d'une incitation scolaire : littérature du début du $\mathrm{XX}^{\mathrm{e}}$ siècle (avant 1960), littérature du XIX ${ }^{\mathrm{e}}$ siècle et du XVIII ${ }^{\mathrm{e}}$ siècle, textes du MoyenAge et de l'Antiquité. 
On perçoit donc un conformisme certain en termes d'écoute de radio et un impact fort des médias sur les goûts musicaux des élèves de cinquième, probablement relayés par les jeunes euxmêmes. Ainsi, Marianne (LP22, parents ouvriers), qui affirme à 17 ans une préférence nette pour le rock, explique :

- J'ai eu une période d'égarement vers des trucs électro... Au début du collège, mais trop de business, c'est pas de la musique ce truc!!!

- Pourquoi as-tu eu cette période alors?

- Je sais pas, parce que c'était la mode, les copains de classe écoutaient ça, alors je faisais pareil, victime de la mode!

Nous avons essayé de mesurer cet effet des influences dans la classe d'école ${ }^{23}$.

Au niveau des goûts musicaux, il existe bien un effet d'entraînement entre élèves d'une même classe, mais il est assez faible : ce ne sont que $30 \%$ (six classes) des classes de cinquième où se lit une influence sur les artistes et groupes cités par les élèves égale ou supérieure à $10 \%$ des élèves de la classe. Par contre, on voit clairement apparaître de mêmes goûts pour l'ensemble des classes de cinquième. Au vu de l'importance de l'écoute de la radio (qui se lit autant dans les résultats de l'enquête par questionnaires que dans les entretiens), on peut soutenir l'hypothèse que la réception médiatique est relayée par l'ensemble des adolescents et que l'influence que les élèves de la classe exercent entre eux n'est que l'expression de ce phénomène plus général.

Au niveau des choix de lecture, le rôle des enseignants est faible puisque les œuvres citées correspondent peu aux programmes ou aux genres légitimes qu'ils défendent. On trouve par contre un effet d'influence dans la classe qui touche sept d'entre elles (35\% des classes de cinquièmes), avec des pourcentages témoignant d'une influence parfois considérable (évoluant entre $20 \%$ et

22 Le type d'établissement où les élèves font leur scolarité est abrégé dans les parenthèses indicatives : LP pour lycée professionnel, LG pour lycée général.

23 Lorsqu'un lien statistiquement significatif ressort, il montre qu'il existe une relation entre le fait d'appartenir à une classe précise et d'avoir tel ou tel goût, mais il ne donne pas le facteur d'influence à l'origine de ce goût. Les élèves d'une même classe peuvent s'influencer mutuellement, mais aussi être influencés ensemble par un ou des enseignants, par exemple. 
$71 \%$ ) entre élèves. Prenons l'exemple d'une classe, où, parmi les 24 élèves, 12 sont des lecteurs de Twilight ${ }^{24}$. La classe se compose de 15 filles et de 9 garçons. Parmi eux, ce sont 11 filles qui ont lu Twilight et un garçon seulement. On perçoit là la forte détermination sexuée du goût que l'on retrouve plus généralement chez les élèves de cinquième avec des lectures assez distinctes (fantasy pour les garçons, littérature féminine de jeunesse, chick lit ${ }^{25}$ et ouvrages de Stephenie Meyer pour les filles) et des goûts musicaux bien répartis (rap pour les garçons et R'n'B pour les filles).

En classe de troisième, on l'a dit, la dispersion des intérêts est plus forte, mais on trouve une influence de la classe sur la lecture dans cinq d'entre elles (33\% des classes de troisième). Les pourcentages d'élèves influencés sont cependant plus faibles et on voit apparaître, de manière transversale et sans répercussion directe au sein de la classe (hormis pour une), littérature contemporaine et littérature d'origine scolaire dont l'immiscion dans les intérêts des élèves est liée à la pression scolaire et à l'avancée dans les programmes. Les entretiens corroborent ce point, nombre des élèves interrogés disant ne plus lire par plaisir, mais par obligation. Par exemple, Laetitia, quand on lui demande pourquoi elle ne lit plus autant qu'avant, répond : "Ben, à cause des livres qu'on doit lire avec le lycée qui font que j'ai pas le temps d'en lire d'autres " (LG, père ouvrier, mère employée).

On voit donc, au niveau des classes de cinquième, un certain conformisme des goûts qui tend à diminuer en troisième, avec l'augmentation de la pression scolaire au niveau de la lecture et la diversification des goûts musicaux.

$\overline{24}$ Twilight, cycle de romans de Stephenie Meyer, raconte la relation amoureuse entre une humaine et un vampire. S. Meyer est l'auteure la plus lue dans notre échantillon.

25 La "chick lit " est un genre récent qui désigne des romans écrits par des femmes et pour des femmes, se caractérisant d'abord par son point de vue, puisque l'histoire est souvent celle d'une jeune citadine, généralement blanche, célibataire et branchée, qui recherche l'amour et le succès professionnel. 


\section{La complexification des influences au lycée}

La diversification des goûts qu'on observe à partir de 14-15 ans et qui s'affirme avec force à partir de 16 ans est clairement liée à l'intensification des sociabilités.

Ainsi, au niveau de l'écoute musicale, c'est $93 \%$ des élèves qui aiment quatre styles et plus qui échangent des titres avec leurs amis alors que ce n'est le cas que pour $78 \%$ des élèves ayant un seul style préféré. On observe des séparations fortes dans les goûts des lycéens en fonction de la filière : là où, pour les élèves de lycée professionnel, apparaissent des prédilections pour le rap, la dance et les musiques du monde, les élèves de première générale et technologique préferent le rock, le folk et la variété francophone.

De même, au niveau de la lecture, en fonction du parcours scolaire, les influences évoluent. C'est ce que montre le tableau suivant.

Tableau 2

Raisons de choix d'un livre en fonction du niveau scolaire

\begin{tabular}{|c|c|c|c|c|}
\hline $\begin{array}{c}\text { Raisons du choix } \\
\text { d'un livre (\%) }\end{array}$ & Cinquième & Troisième & $\begin{array}{c}\text { Première } \\
\text { GT }\end{array}$ & $\begin{array}{c}\text { Lycée } \\
\text { professionnel }\end{array}$ \\
\hline Intérêt personnel & 19 & 18 & 19 & 23 \\
\hline Professeurs & 16 & 16 & 15 & 17 \\
\hline Amis & 13 & 16 & 17 & 15 \\
\hline Hasard & 16 & 14,5 & 13 & 9 \\
\hline Parents & 16 & 12,5 & 12 & 10 \\
\hline Médias & 19 & 23 & 24 & 25 \\
\hline Divers & 1 & 0 & 0 & 0 \\
\hline Total & 100 & 100 & 100 & 100 \\
\hline
\end{tabular}

Question à choix multiples, pourcentage donné sur le nombre de réponses $(\mathbf{n}=$ 2879), taux de non-réponse : 6,6 \%. Lecture du tableau : $19 \%$ des réponses des élèves de cinquième sur les raisons de choix d'un livre indiquent l'intérêt personnel. 
Si les élèves de cinquième sont clairement plus influencés par leurs parents et par le hasard (ce qui évoque clairement, dans ce second cas, le fait que la lecture n'est pas liée aux intérêts scolaires), on observe la montée de l'influence médiatique à partir de la troisième (un peu à l'opposé de ce qui se passe pour la musique) et un poids plus important des amis en première générale et technologique. Ce dernier point mérite d'être souligné, car il est en lien direct avec la sélection scolaire ${ }^{26}$. Ce sont avant tout les élèves n'ayant lu aucun livre dans le dernier mois, les non-lecteurs et les petits lecteurs qui disent suivre les conseils de leurs enseignants pour choisir un livre. Plus on lit, moins on recourt aux conseils des enseignants : de fait, leur influence est plus importante en filière professionnelle où les faibles lecteurs sont plus nombreux, alors qu'en première générale et technologique les amis sont plus présents dans les choix des élèves ${ }^{27}$.

Au niveau de l'écoute musicale, l'influence des médias diffère en fonction de la filière. Les trois radios les plus citées par les élèves (NRJ, Skyrok et Fun Radio) représentent $81 \%$ des choix des élèves de lycée professionnel; elles ne représentent que $63 \%$ des choix des élèves de première générale et technologique, lesquels écoutent davantage les radios musicales pop rock (22\%) et les radios généralistes nationales (7\%). Les goûts de ces élèves se séparent, indépendamment de leur âge, et on peut donc postuler une influence des médias en la matière. Est-ce à dire que le poids du groupe classe est moindre?

Au niveau de l'écoute musicale, au lycée, l'influence au sein de la classe ne se situe plus dans le choix des artistes et des groupes, mais au niveau des genres écoutés, ce qui semble montrer une

26 Nous avons choisi ici de nous baser sur le livre, support de lecture le plus légitime. Mais les résultats sont bien différents pour le choix d'une revue où, en cinquième, le poids médiatique apparaît fortement, alors qu'en première générale et technologique, intérêt personnel, parents et professeurs dominent; ou pour celui d'une bande dessinée, où prédominent l'intérêt personnel en cinquième, les médias et les enseignants en troisième, les amis en première générale et technologique.

27 Nos résultats s'accordent à ceux de Pierre Perier, " La Lecture à l'épreuve de l'adolescence : le rôle des CDI des collèges et lycées ", Revue française de pédagogie, $\mathrm{n}^{\circ} 158$, janvier-mars 2007, p. 43-57. 
plus grande liberté de choix, dans un cadre cependant défini. L'impact des sociabilités juvéniles au sein de la classe est bien plus présent dans les classes de première générale et technologique. Dans $50 \%$ de ces classes, on trouve des pourcentages de goûts communs significatifs (plus de $25 \%$ des élèves apprécient le même genre musical), contre $40 \%$ des classes de LP. Mais on notera que les amateurs d'un même genre musical ne dépassent jamais la moitié de l'effectif total. Cette influence est donc mesurée et laisse des marges importantes de choix. On peut en partie expliquer ce phénomène par la répartition sexuée au sein des classes, comme le montre le tableau suivant.

Tableau 3

Influence du groupe classe sur le choix du genre musical favori

\begin{tabular}{|c|c|c|c|c|c|}
\hline \multirow{2}{*}{ Classe } & \multirow{2}{*}{ Filière } & \multirow{2}{*}{$\begin{array}{c}\% \\
\text { d'amateurs }\end{array}$} & \multirow{2}{*}{$\begin{array}{c}\text { Genre } \\
\text { musical }\end{array}$} & \multicolumn{2}{|c|}{$\begin{array}{c}\text { Pourcentage filles /garçons } \\
\text { aimant le genre musical dans } \\
\text { la classe }\end{array}$} \\
\cline { 5 - 6 } & & & Filles & Garçons \\
\hline 33 & LGT & $42 \%$ & Rock & $47 \%$ & $35 \%$ \\
\hline 35 & LGT & $38 \%$ & Rock & $37 \%$ & 0 \\
\hline 36 & LGT & $37,5 \%$ & Rock & 37,5 & 0 \\
\hline 37 & LGT & $45 \%$ & Rock & $45 \%$ & $45 \%$ \\
\hline 39 & LGT & $40 \%$ & Rock & $32 \%$ & $50 \%$ \\
\hline 21 & LP & $50 \%$ & Rap & 0 & $50 \%$ \\
\hline 46 & LP & $40 \%$ & Rap & 0 & $40 \%$ \\
\hline
\end{tabular}

Lecture du tableau : dans la classe de lycée général et technologique 33 , le pourcentage d'amateurs de rock est de $42 \%$, et ce sont $47 \%$ des filles qui disent aimer cette musique et seulement $35 \%$ des garçons.

En lycée professionnel, deux classes sont uniquement composées de garçons et une proportion conséquente d'entre eux (40 et $50 \%$ ) ont cité le rap comme genre musical favori. De même, en lycée général et technologique, deux classes sont uniquement composées de filles parmi lesquelles on trouve une proportion conséquente d'amateurs de musique rock (38,0 et 37,5\%). 
Restent trois classes mixtes de lycée général et technologique où l'on trouve un pourcentage significatif d'amateurs d'une même musique, le rock. Pour deux d'entre elles, le pourcentage d'amateurs se situe bien plutôt du côté des garçons (classe 39) ou du côté des filles (classe 33). Reste une classe où la répartition d'amateurs d'une même musique se partage équitablement entre garçons et filles (classe 37), indépendamment de la répartition sexuée dans la classe. On voit donc qu'à la fois les goûts sont clairement sexués, mais aussi que la mixité n'est pas suffisante pour expliquer l'influence de la classe sur les goûts individuels : dans les quatre cas où il n'y a pas de mixité, la proportion de personnes appréciant un même genre ne dépasse pas $50 \%$.

Cependant, la "tyrannie de la majorité28 " agit probablement davantage en première générale et technologique qu'en lycée professionnel parce que les élèves y forment un groupe classe plus stable. Deux raisons à cela : d'une part, un certain nombre d'entre eux peuvent parfois avoir suivi une même orientation depuis le collège, d'autre part, le choix des filières et des options permet de disposer d'affinités de base sur lesquelles peut se construire une dynamique de classe. Au contraire, les classes de lycée professionnel représentent souvent un conglomérat d'élèves arrivés là par des voies différentes et parfois hésitantes, les effectifs manquent de stabilité ${ }^{29}$ et la présence de stages au cours de l'année contribue à relâcher le contrôle social de la classe ${ }^{30}$. Les entretiens montrent cependant que la cohésion sociale en

28 Dominique Pasquier, op. cit.

29 D'une part, les formations (BEP et ancien bac professionnel) durent seulement deux ans, et entre BEP et bac professionnel, les classes sont complètement recomposées, avec souvent un changement d'établissement; d'autre part les abandons en cours de scolarité sont plus nombreux.

30 Les périodes de formation en entreprise, en BEP, introduisent des ruptures de cinq à huit semaines sur les deux ans, les périodes de formation en milieu professionnel, en bac pro, de seize à dix-huit semaines sur les deux ans du bac professionnel, tel qu'il existait quand les questionnaires ont été passés. En général, les stages durent plusieurs semaines consécutives. On peut ajouter que le bassin de recrutement des lycées professionnels est beaucoup plus étendu que celui des lycées d'enseignement général et technologique; les élèves de lycée professionnel sont davantage internes et ils ont moins la possibilité d'être en contact le week-end et pendant les vacances. 
lycée professionnel peut augmenter quand les élèves sont majoritairement en internat.

L'influence de la classe est limitée, elle ne s'exerce pas sur tous et autorise les goûts non conformes. La classe se présente comme un ensemble à la fois influent et perméable, tissant des liens de cooptation à des niveaux différents, en fonction de la composition des classes et du niveau d'intégration qui y est nécessaire.

D'une part, donc, une influence plus forte de la classe, mais aussi, d'autre part, une plus grande diversité d'influence médiatique, agissent sur les goûts des élèves des premières générales et technologiques. La différence qui existe par rapport aux influences médiatiques pourrait être liée au cursus scolaire en lui-même, de par les éléments de réflexion critique qu'il est susceptible de transmettre, ce qui expliquerait en partie la nette distance de goûts entre cursus généraux et technologiques et cursus professionnels. Mais, en tout état de cause, nos données ne nous permettent pas de le prouver. Quoi qu'il en soit, il y a bien une influence du système scolaire, ne serait-ce qu'au travers de la sélection qu'il produit.

\section{Les transmissions familiales du goût pour la lecture et l'écoute musicale}

Qu'en est-il des transmissions familiales? C'est bien sûr au niveau de la lecture qu'elles se manifestent le plus. $41 \%$ des grands lecteurs - deux livres au moins par mois, ou un livre au moins et quatre autres supports de lecture (BD, magazine, journal) par mois - sont issus de familles très favorisées, $1,5 \%$ de familles défavorisées. C'est probablement d'ores et déjà dans ce premier rapport à l'écrit que l'influence scolaire trouvera plus ou moins matière à se développer. Les élèves de lycée général et technologique sont plus nombreux à avoir lu un livre dans le dernier mois (76\% d'entre eux) et les élèves de lycée professionnel à n'en avoir lu aucun (58\% d'entre eux). Mais ce n'est là que le résultat de la sélection scolaire dont on sait qu'elle se base sur la maîtrise de l'écrit ${ }^{31}$. Les élèves de lycée professionnel sont les plus nombreux

31 Bernard Lahire, La Raison scolaire. École et pratiques d'écriture, entre savoir et 
à ne pas posséder de livres; ils empruntent moins d'ouvrages et en ont moins acheté que les élèves de lycée général et technologique. $11 \%$ de ces élèves sont des non-lecteurs (ayant déclaré n'avoir rien lu ou n'ayant pas répondu et ne possédant ni livre, ni bande dessinée), contre $0,4 \%$ des élèves de lycée général et technologique. La lecture de livres est incontestablement beaucoup plus présente chez les élèves de lycée général et technologique qui sont aussi les plus nombreux à lire de la littérature d'origine scolaire et à lire la presse nationale. Tous ces éléments indiquent que les choix des élèves de première générale et technologique sont plus marqués par la scolarité. Cependant, cette influence est antérieure à la classe de première : les élèves qui lisent le plus (élèves de première générale et technologique et filles) se laissent davantage conseiller par leurs amis dans leurs choix de lecture. Leurs goûts se sont donc formés, en partie au moins, au contact de l'institution scolaire, puis ils se sont autonomisés, s’associant davantage aux sociabilités juvéniles.

Le rapport familial à la lecture se lit clairement dans les entretiens des jeunes lecteurs. Le plus souvent, c'est la mise à disposition de livres qui agit sur le développement d'un terreau favorable au goût pour la chose écrite. Mais au-delà de cette sensibilisation plutôt passive de la part des parents, l'implication parentale, surtout maternelle, accroît l'intérêt des enfants. Échanges de livres et discussions autour des ouvrages sont souvent présents. On trouvera un autre effet de l'influence parentale sur la lecture à travers le développement d'intérêts communs que la lecture vient simplement soutenir. C'est le cas pour Jean-Paul (LG, parents professions intermédiaires) qui lit la presse sportive et des magazines de moto, intérêt qu'il tient de son père ou pour Marianne (LP, parents ouvriers), qui lit essentiellement des ouvrages consacrés au rock, passion transmise par son père. Dans tous les cas, les conditions d'une sensibilisation efficace à la lecture semblent passer par des activités concrètes, directement ou indirectement liées à la chose écrite. C'est ce que nous appellerons "sensibilisation active à la lecture ". 
La familiarisation à la lecture est clairement associée aux classes moyennes et supérieures. Ainsi, si l'on adopte la classification habituelle en ce qui concerne l'influence des PCS sur la réussite scolaire $^{32}$, parmi les neuf enfants issus de milieux très favorisés ou favorisés, huit ont connu des sensibilisations à la lecture dont la plus grande partie est passive (6/8). Parmi les neuf enfants issus des classes moyennes, cinq ont connu une sensibilisation active à la lecture, un une sensibilisation passive et trois n'en ont connu aucune. Ce n'est qu'un jeune sur les quatre issus des classes défavorisées qui a connu une sensibilisation à la lecture (active).

Il en va autrement pour l'écoute musicale où aucun élément, au niveau quantitatif, ne permet réellement de saisir une influence parentale. Dans les entretiens, par contre, on trouve nombre d'exemples de transmissions directes ${ }^{33}$, liées aux engagements parentaux. Ainsi Marianne (LP, parents ouvriers), dont le père joue dans un groupe de rock local, a hérité de sa passion, et Jean-Paul (LG, parents professions intermédiaires) s'est passionné pour le rock à partir de huit ans après que son père lui a fait écouter un disque. Audrey (LG, père ouvrier, mère employée) a acquis son goût pour le rock par son père et pour la musique classique de sa mère et Natacha (LP, parents employés) dispose de multiples influences familiales. On voit déjà ici des variations dans la manière dont l'intérêt musical s'incarne dans la famille : dans le cas de Jean-Paul ou de Marianne, la figure paternelle est fondatrice, pour Audrey ou Natacha, les passeurs sont plus diversifiés. Dans le premier cas, le goût se transmet à travers un genre précis, dans le second, les intérêts sont beaucoup plus variés.

32 Très favorisée : chefs d'entreprise, cadres et professions intellectuelles supérieures, professeurs des écoles; favorisée : professions intermédiaires (sauf professeurs des écoles), retraités cadres et des professions intermédiaires; moyenne : agriculteurs exploitants, artisans et commerçants (et retraités correspondants), employés; défavorisée : ouvriers, retraités ouvriers et employés, chômeurs n'ayant jamais travaillé, personnes sans activité professionnelle.

33 Il existe aussi une relative sensibilisation, par le fait que les parents écoutent souvent la radio ou de la musique, mais elle apparaît au final peu déterminante, tant la musique imprègne les cultures juvéniles. 
On trouve encore des traces indirectes de l'ascendant parental chez Delphine (LG, parents cadres), dont les influences sont plutôt amicales et médiatiques, mais qui a entendu maintes fois des morceaux que passaient ses parents et qui ont fini par lui plaire, ou chez Julien (LG, père artisan, mère retraitée), ayant de nombreuses sociabilités juvéniles, mais qui, exposé à la musique de sa mère, découvre parfois des perles inattendues parmi des chansons qu'il trouve en majorité datées.

Alors que l'éclectisme musical ${ }^{34}$ est nettement présent parmi les jeunes issus de milieux très favorisés et favorisés dans l'enquête par questionnaires (93\% des jeunes disposant de goûts éclectiques sont issus de ces catégories, ce qui ne représente pourtant que $3 \%$ d'entre eux), il est peu présent au niveau des entretiens où ce n'est qu'un des jeunes qui montre une grande variété de goûts sur les neufs jeunes interrogés. Par contre, on en trouve clairement la trace parmi les jeunes des classes moyennes (4/9). On voit donc bien une confirmation du déploiement des styles musicaux populaires dans l'ensemble de la population, avec probablement une tendance à la transmission des goûts légitimes, faible, mais significative, au niveau des catégories favorisées et très favorisées.

Il est cependant évident ici que l'ascendant familial n'a pas disparu et que la crise de transmission intergénérationnelle n'est pas constante. Qu'en est-il alors de la confrontation avec les sociabilités juvéniles et la scolarité?

\section{Famille, école et sociabilités juvéniles}

En ce qui concerne la lecture, au-delà de l'acquisition de base d'un certain goût pour la lecture dans la famille, on voit les influences scolaires et amicales venir relayer l'intérêt pour la littérature. Les effets de la scolarité sont particulièrement notables, notamment du fait que les jeunes que nous avons interrogés sont au lycée et que les programmes de lecture sont plus chargés qu'au collège. On trouve alors des positions variées qui s'articulent aux

$\overline{34}$ Considéré ici comme l'écoute à la fois de styles populaires (rock, rap, chanson française) et de styles légitimes (musique classique et jazz). 
influences des enseignants et à une plus ou moins grande soumission à l'ordre scolaire.

Sept élèves disent clairement avoir été influencés par un enseignant, que ce soit par des conseils de lecture ou par une pratique concrète (théâtre). Pour la majorité d'entre eux, c'est d'un enseignant précis qu'il est question, et il est souvent décrit par sa capacité à se détacher des programmes scolaires. "En fait, il y avait Madame G. qui nous faisait souvent lire des choses qui sortaient de l'ordinaire. Par exemple, je me souviens d'un roman de Fred Vargas, Debout les morts, qui m'avait vraiment plu et du coup, je me suis intéressé à ses autres livres ", dit Julien (LG, père artisan, mère retraitée).

Pour certains jeunes, l'obligation scolaire apparaît clairement et même nuit à la lecture de loisirs. Par exemple, Laetitia (LG, père ouvrier, mère employée), qui a toujours beaucoup lu, ne se consacre plus qu'aux ouvrages au programme : " c'est quand même dur à lire mais ça m’intéresse, ouais, mais c'est dur à lire, [...] un peu par obligation ".

D’autres enfin sont plus critiques. En général, ils portent un regard négatif sur l'institution scolaire. Ainsi Martine (LP, mère employée) semble déjà avoir abandonné l'idée de finir le diplôme dans lequel elle s'est engagée. Elle se définit elle-même comme une traumatisée de la lecture et trouve la scolarité pénible. Autant dire que les lectures scolaires ne l'inspirent pas.

On le voit, les influences scolaires se construisent de manière positive au travers d'enseignants précis, désignés comme tels par leurs élèves, caractérisés souvent par une moindre recherche de légitimité et une volonté d'impliquer les élèves. La soumission scolaire crée un niveau intermédiaire où il n'est pas sûr qu'on puisse réellement parler d'influence, tant les élèves qui en parlent manquent d'enthousiasme. Une absence d'influence existe aussi, sans pourtant que l'on ne sache dire si elle découle directement de la scolarité ou d'autres facteurs dont, notamment, l'absence de sensibilisation familiale à la lecture.

Les relations entre amis interviennent aussi, de manière assez nette. C'est d'abord le fait que les lectures peuvent faire l'objet 
d'échanges et de discussions entre amis. Paul (LP, parents employés) semble peu intéressé par la lecture en dehors de celle des mangas, qui s'intègre pleinement aux sociabilités de l'internat. Mangas et revues diverses sont des supports de lecture de plaisir qui surgissent souvent en lien avec les sociabilités juvéniles. Ainsi, Julia (LG, parents employés) lit pour son plaisir personnel des revues de mode et des livres de cuisine, mais aussi de la presse people : «avec mes copines, [...] on prend des Ooops!, des Gala et on va dans le parc. On n'achète pas ça parce qu'on aime, mais parce que ça nous fait rire, toutes ces conneries".

La diversité des goûts se lie à la fois à la sensibilisation familiale repérée plus haut et au déploiement des relations sociales. C'est le cas pour Laura (LG, père cadre, mère employée) qui dispose de sociabilités variées, liées à ses différentes activités (communales, sportives, scolaires) et qui lit beaucoup avec d'un côté un goût prononcé pour la fantasy et de l'autre pour la littérature contemporaine, activité soutenue à la fois par ses amis et sa famille. De même, pour Natacha (LP, parents employés), les influences familiales s'élargissent à d'autres sphères et ses choix se font de multiples manières : "par la presse, par les médias, par le bouche à oreille, par les professeurs aussi ». Julien (LG, père artisan, mère retraitée) cite fantastique et autobiographie comme ses genres favoris, résultat des influences scolaires et amicales d'une part, mais aussi de son goût personnel ${ }^{35}$.

Au niveau qualitatif, il existe un véritable lien entre la qualité des relations entretenues dans la famille, dans l'établissement scolaire, dans les groupes amicaux et les influences de lecture. En ce qui concerne les livres, l'école et les influences amicales s'ins-

35 Le « hasard ", item souvent cité dans l'enquête quantitative dans la question sur le choix des livres, laisse peu de prise à l'interprétation statistique. Pourtant, il correspond souvent, dans les entretiens, aux capacités des élèves à déceler dans le paratexte d'un ouvrage le plaisir potentiel qu'ils auront à le lire (" Moi en fait je lis beaucoup les livres en fonction de leur couverture, le devant et le derrière et c'est comme un instinct je sais que ça me plaira ou pas ", dit, par exemple, Damien (LG, parents employés). Le fait que le hasard n'intervienne que pour $9 \%$ des raisons de choix d'un livre pour les lycéens en cursus professionnel et pour $13 \%$ pour les lycéens en cursus général et technologique semble appuyer cette hypothèse. 
crivent dans la continuité de la sensibilisation familiale, alors que l'attraction des revues et des Mangas est davantage liée aux influences familiales (fratrie) et amicales. Famille et école restent donc des éléments déterminants de socialisation à la lecture et se lient plus ou moins aux sociabilités juvéniles, selon les cas.

En ce qui concerne l'écoute musicale, l'influence de l'école n'apparaît qu'au travers des regroupements juvéniles qu'elle permet. Il est peu de trace de l'impact des programmes scolaires sur les goûts adolescents. Par contre, comme pour la lecture, la transmission familiale n'exclut pas les influences amicales, qu'elles soutiennent ou contredisent les goûts familiaux. C'est même, semble-t-il, à la fois ce qui permet tant le maintien des sociabilités juvéniles que la durabilité du goût musical.

Ce jeu d'influences se retrouve bien, quand d'autres ascendants viennent s'ajouter à celui de la famille. Par exemple, le père d'Élise (LG, parents chefs d'entreprise) lui fait découvrir des groupes, mais elle est tout aussi influencée par son petit ami ou son frère. L'impact des aînés de la fratrie revient d'ailleurs souvent dans les entretiens. Pour Sylvain (LG, parents employés), relativement détaché des influences médiatiques et juvéniles ("je peux pas écouter ce qui passe à la radio ou ce que j'entends des portables un peu partout, ça je suis incapable de les écouter ") et très proche de ses parents, cette influence joue un rôle important. Mais c'est le cas aussi pour Laetitia (LG, parents ouvriers) qui, au-delà de son frère, dit découvrir aussi des morceaux par la radio ou la télévision, sa professeure de danse ou ses amis, ou encore pour Nicolas (LG, parents professions intellectuelles supérieures), dont les goûts se construisent au confluent de ses amitiés, des conseils de son professeur de batterie et de sa sœur. Ici, de multiples influences se déploient sans que l'une ou l'autre prenne vraiment l'avantage.

Un autre modèle se détache, où la prédominance des sociabilités juvéniles s'articule à un relatif retrait des relations familiales. C'est le cas pour Bernard (LP, père ouvrier, mère au foyer) qui n'écoute que du rap et dont les goûts sont essentiellement liés au groupe d'amis avec lequel il passe énormément de temps, mais 
aussi pour Martine (LP, mère employée) qui ne trouve de plaisir que dans des sociabilités qui se construisent en grande part sur la musique qu'elle écoute ou joue.

La distance ne s'affirme pas seulement par la froideur des relations, mais plus simplement par l'âge. Ainsi, Julien (LG, père artisan, mère retraitée) dit de ses parents qu'ils sont vieux et trouve la musique qu'ils écoutent inintéressante.

L'internat est un autre facteur de distanciation possible. Il agit comme une prise de recul vis-à-vis des influences familiales quand les jeunes gens immergés dans ce nouveau milieu s'y sentent bien et s'y font de nouveaux amis. C'est le cas pour Vincent (LG, parents professions intermédiaires) ou pour Paul (LP, parents employés). Ils illustrent bien l'influence des filières scolaires que nous avait révélée l'enquête quantitative, puisqu'alors que Vincent se tourne vers le rock, Paul préfere le rap et la pop, qu'il écoute avec ses copains, le soir, à l'internat. Vincent explique bien ce phénomène :

Et puis après on est potes, mais on a à peu près les mêmes idées, donc on écoute tous à peu près la même chose, Albert et tout, on est vachement rock, et heu, plus il y a de potes qui arrivent, plus on apprend des nouveaux groupes, des trucs comme ça. [...] Mais, heu, ouais, parce qu'on voit plus, une fois qu'on est interne, on voit plus ses potes que sa propre famille, vu qu'on les voit que le week-end, forcément, ça resserre vachement, quoi, enfin moi, ça me fait ça en tout cas...

Les préférences musicales sont aussi liées à la constance des liens amicaux. On a vu plus haut l'exemple de Marianne (LP, parents ouvriers) qui confessait avoir écouté, sous l'influence de ses camarades de collège, une musique qu'elle trouve désormais commerciale. De même Vincent (LG, parents professions intermédiaires) avoue avoir écouté du métal avec un ami qu'il voit beaucoup moins.

Si l'influence scolaire est absente des propos des jeunes, on observe des variations dans l'impact des sociabilités juvéniles, en fonction des relations entretenues dans la famille, qu'il y ait une passion d'un ou plusieurs parents pour la musique ou non. Le rôle potentiel de la famille se lit ainsi parfois en creux, dans la place qu'elle laisse aux sociabilités juvéniles. 


\section{Goûts et configurations relationnelles}

Les données qualitatives font évoluer notre regard vers des configurations de relations sociales ${ }^{36}$, qui, entre transmission familiale, déploiement des sociabilités juvéniles et insertion scolaire, développent des tendances à se laisser influencer par certains agents plutôt que par d'autres. La compréhension des modes d'articulation de ces influences est alors primordiale. Ils differrent cependant selon que l'on considère l'écoute musicale ou la lecture.

Au niveau musical, les transmissions familiales sont bien présentes. Elles s'établissent en fonction de l'intérêt des parents pour la musique et de la qualité des liens familiaux. Les influences médiatiques directes semblent faibles; elles sont souvent instrumentalisées par les jeunes qui s'en servent pour nourrir leur besoin d'intégration ou d'affirmation dans les groupes de pairs. Les sociabilités juvéniles comportent donc bien une dimension normative qui oblige certains jeunes à "se tenir au courant ", sans pourtant les contraindre totalement dans leurs goûts.

Plusieurs configurations d'influences apparaissent alors.

D'une part, on voit des jeunes dont le goût musical a été fortement affecté par la famille, qu'il s'agisse des parents ou des frères et sœurs, et se trouve relayé par des sociabilités juvéniles conséquentes ( 7 personnes), d'autre part des jeunes en retrait de leurs familles pour différentes raisons (différence d'âge, internat, relations distantes) mais disposant de sociabilités juvéniles fortes qui agissent sur leurs goûts (6 personnes). Entre les deux, se trouvent des jeunes à qui les membres de leur famille n'ont pas

36 On entendra ici le terme de " configuration » dans un sens proche de celui développé par Norbert Élias : un système d'interdépendances qui relie différentes personnes et où "s'établit un équilibre fluctuant des tensions, un mouvement pendulaire d'équilibre des forces, qui incline tantôt d'un côté, tantôt de l'autre ». Norbert Élias, Qu'est-ce que la sociologie, La tour d'Aigues, Éditions de l'Aube, 1991, p. 158. Élias précise que le concept de configuration peut aussi bien s'appliquer à des groupes restreints qu'à des sociétés. On notera que, si nous abordons dans cet article davantage l'échelle des groupes restreints, l'enquête par questionnaire montre l'impact du marché des biens culturels, à travers, par exemple, la prédominance forte des œuvres anglosaxonnes parmi les livres lus et les musiques écoutées dans notre échantillon. 
transmis de goût particulier, mais qui entretiennent des relations soutenues avec des jeunes de leur âge (6 personnes).

Ces trois configurations permettent d'expliquer les goûts des jeunes pour $86 \%$ de notre échantillon (22 personnes). Si le rôle des médias n'est pas à négliger, il est ici périphérique et dépend plutôt des logiques de différenciation ou d'homogénéisation qui vont permettre aux jeunes de se situer dans les groupes amicaux et les familles : il est utilisé en fonction de ce que recherchent les jeunes. L'impact des sociabilités juvéniles est plus important que celui des familles, puisque leur influence ne semble périphérique que pour trois personnes. Mais l'influence des pairs se définit en fonction de la transmission musicale opérée ou non par les familles et de la qualité et de l'intensité des relations entretenues avec les autres jeunes ${ }^{37}$.

On notera enfin que ce ne sont que $2 \%$ des jeunes de l'enquête quantitative et cinq des jeunes interrogés qui disposent d'un véritable éclectisme ${ }^{38}$ au niveau des goûts musicaux, ce qui soutient l'hypothèse d'Hervé Glevarec d'un goût majoritairement populaire des jeunes en matière de musique.

Du côté de la lecture, on pourrait résumer le rôle du milieu familial ainsi : une sensibilisation à la lecture active et impliquée produit une relative disponibilité des enfants aux œuvres inscrites dans les programmes, un bon climat familial - sans sensibilisation particulière à la lecture - tend à entraîner une soumission à l'ordre scolaire, qui peut ou non permettre l'accès aux œuvres, un désintérêt familial, de mauvaises relations entre parents et enfants ou encore une soumission trop forte aux sociabilités juvéniles (ces différents éléments pouvant bien sûr s'associer) amènent plus facilement à un rejet de la lecture, souvent associé à un rejet de la scolarité.

Parmi les huit jeunes qui ont parlé à un titre ou un autre de rencontres significatives avec la lecture par l'intermédiaire d'un

\footnotetext{
37 Il est important de mesurer ici les deux critères. Dans certains cas, la diminution des relations familiales (intensité) n'empêche pas que celles-ci restent prégnantes (qualité). (musique classique ou jazz) dans les goûts des élèves.
} 
enseignant, six avaient été activement sensibilisés à la lecture par leur famille. C'est au demeurant ce premier trait qui permet d'ébaucher, là encore, des configurations relationnelles, associant famille et école. On voit clairement que la socialisation familiale active à la lecture agit directement sur la réception des œuvres scolaires, de même qu'un bon climat familial favorise l'acceptation de la nécessité de lire les ouvrages scolaires (7 personnes) et que l'éloignement entre jeunes et familles agit sur la réception des lectures scolaires ( 3 personnes). Ces trois configurations cumulent $73 \%$ des situations.

Ce qui apparaît le plus déterminant alors, ce n'est pas tant le rôle des agents majeurs de socialisation (d'un côté l'école ou la famille, de l'autre les groupes de pairs) que les configurations relationnelles dans lesquelles ils agissent.

Ces configurations agissent d'ailleurs parfois aussi bien sur le goût pour la lecture que sur le goût pour la musique. Les situations de Sylvain (LG, parents employés) ou de Benjamin (LG parents retraités de l'enseignement) le montrent bien. Tous deux sont très proches de leurs familles et sont aussi de grands lecteurs de fantasy. Tous deux ont une implication faible dans les sociabilités juvéniles scolaires et des activités périscolaires importantes. Alors que Sylvain est un bon élève, assumant sa distance aux stéréotypes culturels de ses camarades tant sur le plan musical que sur le plan de la lecture (en début d'entretien, il affirme n'être pas très représentatif des jeunes de son âge en disant : " déjà, je lis...»), Benjamin a des choix musicaux très proches de ses camarades (rap et reggae) et voit ses résultats scolaires baisser. Dans un cas, le relatif écartement des sociabilités juvéniles permet d'assumer des goûts distinctifs clairs, dans l'autre, il oblige à se conformer aux goûts de ses camarades. Différence assumée et recherche d'intégration se lient ici pour proposer deux situations symétriquement inverses.

On trouve un même modèle dans l'insertion dans les groupes qui se forment en milieu scolaire : l'influence des pairs est essentiellement liée à la cohésion sociale dans la classe ou le groupe d'amis. Plus l'on se sent intégré à un groupe, plus l'on 
partage avec ce groupe des goûts et plus ces goûts acquièrent une valeur distinctive. Les exemples de Marianne et Vincent, tous deux internes, le montrent bien.

Nous avons vu plus haut que l'arrivée de Vincent (LG, parents professions intermédiaires) à l'internat d'un établissement de milieu rural menait à l'émergence d'amitiés fortes et influentes, qui agissent directement sur ses loisirs. Parallèlement, on observe une baisse sérieuse de ses résultats, alors même qu'il caresse l'idée de devenir enseignant. Son goût pour la lecture est faible, même si, en digne fils de professeur des écoles, il dit savoir " qu'il y a de très bons livres".

Pour Marianne (LP, parents ouvriers), la configuration est toute différente. Son insertion dans l'internat du lycée professionnel où elle se trouve ne lui a pas apporté de nouvelles amitiés. Le soir, elle appelle son petit-ami et parfois ses parents.

Au lycée, je partage pas ma passion pour le rock avec les autres... Sinon j'ai des copines, je leur parle, mais sans plus... je suis très seule, solitaire et j'ai besoin de cette musique pour rendre les journées plus agréables... À X (son village d'origine), avec mes amis, on partage les mêmes goûts musicaux, donc c'est mieux...

On comprend bien que son ancrage musical, à la fois familial et amical, est quelque peu exclusif et ne laisse pas beaucoup de place à des goûts nouveaux.

\section{L'intégration dans les groupes : conformisme et différenciation}

Ce que ces configurations nous dévoilent aussi, c'est que le goût pour la musique ou pour la lecture peut être aussi bien utilisé comme facteur d'affirmation de soi que d'intégration. La lecture, certes, sert davantage à se différencier dans les relations avec les adultes (famille, école) que dans les sociabilités juvéniles, même si cela est parfois le cas dans quelques groupes restreints.

Au contraire, la nécessité de s'insérer dans les groupes est bien à la base de consommations médiatiques, qui permettent aux jeunes de savoir ce qui se passe dans l'actualité musicale juvénile. On trouve ainsi des jeunes qui regardent les clips à la télévision 
ou écoutent la radio "pour se tenir au courant ", comme Laetitia (LG, parents ouvriers) ou Bertrand (LG, père cadre, mère retraitée). Pour Julia (LG, parents employés), jeune fille très impliquée dans la mode qui lui sert à affirmer sa personnalité, la musique - qu'elle écoute pourtant beaucoup - n'est clairement pas un moyen de distinction : "il y a des gens qui écoutent des choses que personne ne connaît, ils doivent trouver ces chanteurs ou ces groupes sur Internet, je pense. Moi, j'avoue que ça m'intéresse pas tellement, si une chanson passe à la radio et que j'aime, alors je me la procure ». On note qu' elle est fidèle à deux des trois radios grand public les plus écoutées par les jeunes de l'échantillon quantitatif.

Dans d'autres cas, plus souvent masculins, l'écoute musicale apparaît comme un outil de différenciation, associé de manière nette à la valorisation de l'originalité de la personnalité et parfois au mépris des productions commerciales. C'est particulièrement net dans l'entretien avec Julien (LG, père artisan, mère retraitée) : "j'écoute un peu de tout, mais c'est vrai que j'aime bien les trucs que personne connaît. Ça me parle. [...] Mais c'est cool aussi quand quelqu'un fouille dans ton portable et qu'il trouve une musique qu'il ne connaît pas ».

Différenciation et insertion ne s'opposent pas nécessairement. On voit ainsi des jeunes qui ont des goûts assez éclectiques passer d'un registre à un autre. C'est le cas de Damien (LG, parents employés), dont les goûts se distinguent de ses amis comme de sa famille (il a décliné aussi bien l'offre de ses copains que celle de ses parents d'aller voir des concerts qui ne l'attiraient pas). S'il aime le rock indépendant, il dit écouter NRJ " pour être au courant des hits en ce moment ". On voit là une tension qui lui rend nécessaire une prise sur l'actualité musicale juvénile, alors même que ses goûts se distinguent de ceux de ses proches.

Enfin, il faut noter que l'éclectisme semble être une fonction directe de la variété des réseaux de relations entretenus. Cela se lit clairement chez Natacha (LP, parents employés), Virginie (LG, père commerçant, mère employée), Delphine (LG, parents cadres 
et professions intellectuelles supérieures) et quelques autres. Cependant, le déploiement de réseaux en lien avec l'éclectisme semble être une caractéristique plutôt féminine.

\section{Conclusion}

La variable explicative qui se détache le plus clairement est bien le rattachement à des sociabilités et le niveau de cohésion qui les fonde. Il faut davantage voir l'influence des groupes de pairs comme un système d'affinités électives que comme une tyrannie de la majorité, même si, en établissement, comme on l'a vu par l'enquête quantitative, une influence du groupe classe est bien présente.

Les influences varient en fonction du niveau de cohésion interne, que ce soit dans la famille, la classe ou les groupes de pairs. Il existe bien des degrés de liberté par rapport aux groupes d'appartenance, selon la force des attachements. Quand une influence s'exerce davantage sur les adolescents, c'est parce que certains goûts sont plus nécessaires que d'autres à l'intégration dans un groupe, quel qu'il soit, et donc que ces goûts classent les personnes dans le groupe. Des normes émergent et se réifient lorsqu'un groupe est suffisamment stable; ceux qui en font partie ou veulent en faire partie vont alors y adhérer, de manière plus ou moins volontaire. C'est aussi bien le cas dans les familles que dans les classes et les groupes de pairs. La contrainte apparaît pourtant peu, sinon dans l'obligation que se donnent quelquesuns de "se tenir au courant ». Les influences sont acceptées plutôt que subies.

Des classements s'opèrent donc, à l'intérieur des groupes et entre les groupes, et les goûts ne sont pas si relatifs ${ }^{39}$. Cependant, les parcours de socialisation sont complexes et soumis à des influences diversifiées, qui varient en fonction des domaines d'intérêt. Ainsi, si la scolarité agit sur la propension à lire et à lire des choses plus diverses pour la plupart des élèves, elle prend

39 "Le goût est une disposition acquise à "différencier" et "apprécier", comme dit Kant, ou, si l'on préfère, à établir ou à marquer des différences par une opération de distinction [...] ", Pierre Bourdieu, La Distinction, op. cit., p. 543. 
corps dans le prolongement des sensibilisations à la lecture dans la famille. Pour les élèves de lycée professionnel qui ont peu connu cette sensibilisation, les impulsions du groupe classe ont plus d'effet. Au contraire, ce dernier agit davantage sur les choix musicaux des élèves en lycée général et technologique, alors que l'emprise des médias, et notamment de la radio, est plus importante pour les élèves de lycée professionnel. En aucun cas, il n'existe de mainmise des groupes de pairs sur les intérêts culturels des élèves ${ }^{40}$.

L'influence des parents dépend du climat familial, celle des enseignants du rapport à l'école, celle des amis de la nécessité de s'intégrer à un groupe et tous ces contextes s'imbriquent et se définissent mutuellement. Pourtant la socialisation parentale reste à la base du développement des goûts, dans la mesure où elle permet (ou non) des prolongements dans le domaine des amitiés juvéniles ou des intérêts scolaires. C'est pourquoi l'approche bourdieusienne n'est pas invalidée : le poids des origines sociales reste un facteur explicatif fort.

Par contre, le processus de socialisation s'est complexifié, de par la multiplication des réseaux et groupes auxquels appartiennent les adolescents. Cette complexification, en retour, produit une autonomisation relative des supports mobilisés pour se distinguer, par rapport à la définition des biens légitimes au niveau institutionnel. La hiérarchisation classique de la légitimité sociale s'applique moins et les cultures populaires dominent les intérêts juvéniles. De même, l'opposition entre éclectisme et exclusivité, classes supérieures et classes populaires se lit assez peu au niveau de l'écoute musicale comme au niveau de la lecture (contrainte par les programmes scolaires et donc assez générale). Il ne faudrait pas, pourtant, confondre les influences et leurs contextes d'émergence. Ce que nous montrent les entretiens, c'est que les goûts naissent aussi d'occasions, parfois répétées (écouter la station de radio choisie par le chauffeur du bus de ramassage

40 On retrouve cette même complexité dans d'autres domaines, notamment dans l'acquisition des compétences numériques : ce n'est pas dans la simple opposition entre les domaines de la lecture et des goûts musicaux que sont présents des arbitrages fins entre les différents agents d'influence. 
scolaire ou par ses parents, faire de la danse et découvrir une série de littérature jeunesse consacrée à ce milieu, etc.), parfois isolées (une musique entendue à la radio, un livre feuilleté), parfois s'inscrivant dans un contexte plus complexe où la révélation s'articule à un ascendant précis (une enseignante qui conseille un livre inattendu, un père qui passe de la musique dans la voiture). Des arbitrages subtils modifient les goûts (ne plus écouter un style musical parce qu'on a perdu de vue l'ami avec qui on l'écoutait, lire davantage parce que l'on a plus d'argent de poche).

Qu'apparaissent des agencements variés et variables en fonction des domaines considérés ne peut nous étonner : les adolescents ne réagissent pas avec la même plasticité aux suggestions des producteurs de normes culturelles selon leurs parcours familiaux, leurs histoires scolaires, leurs sociabilités, la structuration et la dynamique de la classe. Sensibilité au conformisme et diversification des goûts ne s'opposent donc pas, mais s'affirment différemment en fonction de ces variables.

C'est dans l'action conjointe des situations familiales et des réseaux relationnels, des activités de loisirs et des parcours scolaires que semblent se créer des zones d'influence plus ou moins puissantes, déterminées en grande partie par la cohésion des différentes instances en présence. Ainsi, la qualité des relations avec les parents, les modes de scolarisation (internat ou externat), les filières et la cohésion interne des groupes classes sont des facteurs d'explication d'une emprise plus ou moins durable sur les goûts adolescents. Le conformisme adolescent n'existe alors que par rapport à des configurations qui associent des groupes sociaux (famille, classe, groupes de pairs) en fonction de la qualité et de l'intensité des liens qui y existent. Omnivorité et éclectisme aussi bien que conformité et distinction peuvent prendre sens dans ces configurations d'influences.

Hétérogénéisation et homogénéisation des goûts apparaissent alors comme des vecteurs de production du sens, agissant dans tous les parcours par des opérations de distinction : on se conforme ou l'on s'affirme par les goûts que l'on montre. Plutôt que d'opposer des modèles, il est bien question ici de les associer 
en en mesurant les importances relatives et en essayant de modéliser les effets des attachements aux différents groupes en présence. L'articulation de données qualitatives et quantitatives n'est certes pas nouvelle. Elle est probablement encore trop fragile ici et il reste à amplifier la vague d'entretiens pour produire des résultats plus sûrs, qui renforceront et rendront plus compréhensibles les résultats quantitatifs.

Mais, si, finalement, les oppositions entre courants montrent leur productivité en forçant le renouvellement des appareils conceptuels par le jeu successif des ajustements et réajustements de la pensée, c'est le fait de les considérer aussi valides les uns que les autres qui permet de déboucher sur des articulations plus fines et plus sensibles.

Surtout, les pratiques culturelles semblent produire autant d'hétérogénéité que d'homogénéité, parce qu'une multitude de formations sociales englobent l'individu et que les références qu'il adopte construisent sa singularité autant que sa conformité, en fonction de ses appartenances. C'est l'interaction entre ces formations sociales qu'il reste à explorer pour mieux en comprendre le fonctionnement. À une époque où réseaux et groupes de référence pullulent, où tout individu doit se situer au confluent de multiples influences, se conformer c'est forcément se distinguer; se distinguer, c'est obligatoirement se conformer. Il s'agit là d'un processus auquel aucun d'entre nous n'échappe et qui nous oblige à nous positionner dans des champs relationnels autant que de connaissance ou d'approche scientifique. 


\section{Bibliographie}

Bourdieu, Pierre, «La Spécificité du champ scientifique et les conditions sociales du progrès de la raison ", Sociologie et sociétés, vol. 7, nº 1, 1975, p. 91-118.

Bourdieu, Pierre, La Distinction. Critique du jugement, Paris, Minuit, 1979. Bourdieu, Pierre, Questions de sociologie, Minuit, Paris, 1984.

Buckingham, David, La Mort de l'enfance, grandir à l'âge des médias, Paris, Armand Colin, 2000.

Combessie, Jean-Claude, La Méthode en sociologie, La Découverte, Paris, 2007.

Coulangeon, Philippe, Les Métamorphoses de la distinction, Paris, Grasset, 2011.

Donnat, Olivier (dir.), Regards croisés sur les pratiques culturelles, Paris, La Documentation Française, 2003.

Donnat, Olivier, Les Français face à la culture. De l'exclusion à l'éclectisme, Paris, La Découverte, 1994.

Elias, Norbert, Qu'est-ce que la sociologie?, La tour d'Aigues, Éditions de l'Aube, 1991.

Glevarec, Hervé et Michel Pinet, "La "Tablature” des goûts musicaux : un modèle de structuration des préférences et des jugements ", Revue française de sociologie vol. 50, n ${ }^{\circ} 3,2009$, p. 599-640.

Harris Judith, Pourquoi nos enfants deviennent ce qu'ils sont, Paris, Laffont, 1999.

Lahire, Bernard, La Culture des individus. Dissonnances culturelles et distinction de soi, Paris, La Découverte, 2004.

Lahire, Bernard, La Raison scolaire. École et pratiques d'écriture, entre savoir et pouvoir, Rennes, Presses universitaires de Rennes, 2008.

Lizé, Wenceslas et Olivier Roueff, "La Fabrique des goûts ", Actes de la recherche en sciences sociales, $\mathrm{n}^{\text {os }}$ 181-182, mars 2010, p. 4-11.

Maigret, Éric et Éric Macé (dir.), Penser les médiacultures, Paris, Armand Colin, 2006.

Octobre, Sylvie et al., L'Enfance des loisirs. Trajectoires communes et parcours individuels de la fin de l'enfance à la grande adolescence, Ministère de la Culture, Paris, 2010.

Pasquier, Dominique, Cultures lycéennes, la tyrannie de la majorité, Paris, Autrement, 2005.

Pasquier, Dominique, "La Culture comme activité sociale ", dans Éric Maigret et Éric Macé (dir.), Penser les médiacultures, Paris, Armand Colin, 2006, p. 103-119. 
Perier, Pierre, "La Lecture à l'épreuve de l'adolescence : le rôle des CDI des collèges et lycées ", Revue française de pédagogie, n 158 , janviermars 2007, p. 43-57.

Peterson, Richard A. et Alfred Simkus, "How Musical Tastes Mark Occupationnal Status Groups ", dans Michèle Lamont et Marcel Fournier (dir.), Cultivating Differences, Symbolic Boundaries and the Making of Inequality, Chicago, Londres, University of Chicago Press, 1992, p. 152-186. 